\title{
NUMEROS CROMOSOMICOS EN ALGUNAS ESPECIES DE ASTRAGALUS (FABACEAE) Y UNA ENMIENDA A LA DESCRIPCION DE A. ZACATECANUS (RYDB.) BARNEBY
}

\author{
Richard Spellenberg y Sepgio Rodriguez T. \\ Department of Biology \\ New Mexico State University \\ Las Cruces, NM 88003-0001, U.S.A.
}

\begin{abstract}
RESUMEN
Se dan a conocer los números cromosómicos de 18 taxa de Astragalus (Fabaceae) del norte de México y el suroeste de los Estados Unidos. Los datos correspondientes a las 12 especies siguientes se revelan por primera vez: CONTEOS PROVENIENTES DE BOTONES FLORALES: $(n=11) A$. cyaneus, $A$. eremiticus, $A$. insularis var. harwoodii, $A$. proximus; $(n=14) A$. hartwegii; $(n=15) A$. goldmanii, $A$. zacatecanus; CONTEOS PROVENIENTES DE SEMILLAS: $(2 n=22) A$. cottamii, $A$. holmgreniorum, $A$. humillimus, $A$. wittmanii; $(2 n=28) A$. vaccarum. Seis conteos confirman citas anteriores, a mencionar: CONTEOS PROVENIENTES DE BOTONES FLORALES: $(n=11) A$. cobrensis var. maguirei, $A$. fucatus, $A$. lentiginosus var. variabilis, $A$. longissimus, $A$. mollissimus var. ?; $(n=15) A$. diphacus. Se informa sobre la segunda y tercera colectas y el primer registro para Durango de $A$. zacatecanus, una especie anteriormente conocida solamente del tipo. Se hace una enmienda de la descripcion con base en la morfología de las nuevas colectas. Se incluye una discusión sobre la significación de los números cromosómicos observando que en Norteamérica los números cromosómicos más grandes son caracteristicos de ciertos grupos mexicanos de Astragalus.
\end{abstract}

\section{ABSTRACT}

Chromosome numbers are reported for 18 taxa of Astragalus (Fabaceae) from northern Mexico and the southwestern United States. Twelve are reported for the first time and are as follows: COUNTS FROM BUDS: $(n=11) A$. cyaneus, $A$. eremiticus, $A$. insularis var. harwoodii, $A$. proximus; $(n=14) A$. hartwegii; $(n=15)$ A. goldmanii, $A$. zacatecanus; COUNTS FROM SEEDS: $(2 n=22) A$. cottamii, $A$. holmgreniorum, $A$. humillimus, $A$. wittmanii; $(2 n=28) A$. vaccarum. Six confirm earlier reports and are: COUNTS FROM BUDS: $(n=11) A$. cobrensis var. maguirei, $A$. fucatus, $A$. lentiginosus var. variabilis, A. longissimus, $A$. mollissimus var. ?; $(n=15) A$. diphacus. Second and third records for the species, and a new record for Durango, are reported for $A$. zacatecanus, a species formerly known only from the type collection. An emendation of the description is made based on the morphology of the new collections. A brief discussion regarding the significance of the chromosome numbers is presented, noting that higher chromosome numbers are characteristic for certain Mexican groups of Astragalus.

Se dan a conocer en este trabajo varios números cromosómicos correspondientes a colectas de Astragalus realizadas en el campo. Los cromosomas se observaron por el método de aplastamiento (squash) de botones florales o extremos de raíces en preparaciones elaboradas por métodos convencionales (Spellenberg, 1976). Para los ejemplares de respaldo se señalan los herbarios en que éstos se depositaron (en esta 
lista ESAHE = Escuela Superior de Agricultura "Hermanos Escobar", en Ciudad Juárez, Chihuahua). Aquellos conteos que son los primeros para un taxon determinado se marcan con un asterisco ("), a los provenientes de botones florales corresponde la indicación $n$, y a los provenientes de extremos de raíces $2 \mathrm{n}$. El total comprende conteos de 18 taxa, de los cuales 12 son nuevos registros; todos los números obtenidos para los restantes taxa confirman los conteos publicados anteriormente.

A. cobrensis A. Gray var. maguirei Kearney. $\mathrm{n}=11$. USA, Arizona, Morse Canyon on W side of Chiricahua Mountains, 6 Apr 1980, Spellenberg and Soreng 5448 (NMC). De 152 células en anafase I tardla o telofase I temprana, 5 tenlan vestigios de un puente de anafase $\mathrm{I}$.

A. "cottamii Welsh. $2 \mathrm{n}=22$. USA, New Mexico, San Juan Co., ca. $4 \mathrm{mi}$. SW of Waterflow, 31 May 1985, Spellenberg and Corral D. 8203 (NMC, NY, RSA).

A. "Cyaneus A. Gray. $n=11$. USA, New Mexico, Rio Arriba Co., NW of Embudo, 5 May 1978, Spellenberg 4983 (NMC, NY).

A. diphacus S. Wats. $n=15$. México, Durango, 7 mi. $S$ of Súchil. 22 Jul 1990, Spellenberg and Gonzalez E. 10268 (ESAHE, ID, MEXU, NMC, NY).

A. "eremiticus Sheld. $\mathrm{n}=11$. USA, New Mexico, Hidalgo Co., Riley Peaks near Virden, 6 Apr 1986, Spellenberg et al. 8401 (NMC, NY).

A. fucatus Barneby. $\mathrm{n}=11$. USA, New Mexico, Socorro Co., $13.6 \mathrm{mi}$ of Magdalena, 8 May 1977, Spellenberg 4653 (NMC).

A. "goldmanii M. E. Jones. $\mathrm{n}=15$. México, Chihuahua, $27 \mathrm{~km} \mathrm{~W}$ of Parral on road to Guadalupe y Calvo, 12 Jul 1986, Spellenberg 8534 (ESAHE, MEXU, NMC, NY).

A. "hartwegii Benth. $\mathrm{n}=14$. México, Durango, ca. 60 air km SE of Durango at La Reserva Biosfera "La Michilia", 27 Jul 1990, Spellenberg and Gonzalez E. 10376 (NMC).

A. "holmgreniorum Barneby. $2 \mathrm{n}=22$. USA, Arizona, $13.5 \mathrm{~km}$ airline distance $\mathrm{S}$ of St. George, Utah, 25 May 1979, Holmgren, Holmgren and Barneby 9175. El conteo fue obtenido de semillas provenientes del isotipo en NMC.

A. "humillimus A. Gray ex Brandeg. 2n = 22. USA, New Mexico, San Juan Co., The Hogback, 10 Aug 1986, Spellenberg and Ward 1986 (COLO, K, NMC, NY).

A. "insularis Kell. var. harwoodii Munz \& McBurney ex Munz. $n=11$. México, Sonora, $29 \mathrm{mi}$. by road S of Desemboque, S of Puerto Libertad, 22 Mar 1978, Spellenberg 4949 (NMC).

A. lentiginosus Dougl. var. variabilis Barneby. $\mathrm{n}=11$. USA, California, San Bernardino Co., ca. $1 \mathrm{mi}$. N of Hinckley, 3 Apr 1986, Strother 1348 (NMC, UC). 
A. longissimus (Torr.) Barneby. $\mathrm{n}=11$. México, Chihuahua, Mpio. Ocampo, $4 \mathrm{mi}$. E of Ocampo, 25 Sep 1988, Spellenberg 9748 (NMC, MEXU) (corola azul-púrpura, pálida y ocroleuca, alas dobladas hacia afuera); $2 \mathrm{Z}=22$. Chihuahua, Mpio. Gómez Farías, 9 mi. S of Peña Blanca, 3 Aug 1988, Spellenberg et al. 9666 (MEXU, NMC) (corola de color amarillo claro, alas dobladas hacia adentro en forma de cuchara).

A. mollissimus Torr. var. "?. $\mathrm{n}=11$. México, Chihuahua $27 \mathrm{~km} \mathrm{~W}$ of Parral on road to Guadalupe y Calvo, 12 Jul 1986, Spellenberg 8535 (NMC). La identidad infraespecifica de esta colecta no ha sido aún determinada. Se han realizado varias visitas al sitio de colecta, pero no se ha encontrado suficiente material en fructificación para hacer la determinación. No se han observado corolas amarillas para esta especie en la localidad indicada.

A. "proximus (Rydb.) Woot. \& Standl. $\mathrm{n}=11$. USA, New Mexico, San Juan Co., $4 \mathrm{mi}$. NNE of La Plata, 5 Jun 1984, Spellenberg and Ruzzo 7794 (ASU, ID, NMC, NY).

A. "vaccarum A. Gray. $2 n=28$. USA, New Mexico, Hidalgo Co., Las Playas Springs, 10 Oct 1982. Soreng and Spellenberg 2035 (NMC).

A. "wittmanii Barneby. $2 n=22$. USA, New Mexico, Harding Co., $2 \mathrm{mi} \mathrm{W}$ of Mills. Spellenberg et al. 5991 (COLO, ISC, NMC, NY).

A. "zacatecanus (Rydb.) Barneby. $\mathrm{n}=15$. México, Durango, ca. 47 air km SSW of Vicente Guerrero, in Sierra Urica, 26 Jul 1990, Spellenberg and González E. 10354 (MEXU, NMC, NY). Esta colecta, y otra (Durango, mpio. Súchil, San Juan de Michis, rumbo al Cerro "Chihuahuilla", 14 Aug 1984, F. Chávez 53, CIIDIR, NMC, NY) son aparentemente la tercera y segunda, respectivamente, para la especie y representan una extensión de su distribución conocida hacia el norte de unos 120 kilometros dentro del estado de Durango. La planta se conocía solamente del suroeste de Zacatecas de 1897 (Barneby, 1964). Como es frecuente en las colectas antiguas, el hábitat no se ha registrado en la etiqueta del ejemplar y permaneció desconocido para Barneby al tiempo de su revisión del género. La colecta tipo no tiene frutos maduros, y como era de esperarse, los individuos de esta segunda población difieren ligeramente de los de la primera. Las muestras colectadas por Spellenberg y González E. en Durango provienen de la orilla de un viejo camino maderero en suelo arenoso-arcilloso claro, a una altitud de $2630 \mathrm{~m}$. La población de la orilla del camino se origino aparentemente a partir de plantas que crecian (pero no estaban aún en floración) sobre una ladera descubierta entre pastos perennes amacollados. El bosque, como Barneby lo supuso, era mixto de encino y pino.

En la siguiente ampliación de la descripción de A. zacatecanus, las caracteristicas conocidas por Barneby se indican entre paréntesis. Las plantas de Durango son erectas o fuertemente ascendentes (aparentemente decumbentes); las superficies adaxiales de los folíolos son esparcidamente estrigosas (glabras, pero Barneby (comunicación personal) observó unos pocos tricomas en las superficies adaxiales de los folíolos en el holotipo en NY); cáliz de $5 \mathrm{~mm}$ de longitud (6.1-7 mm); corola verdosa-amarillenta (ocroleuca); estandarte de $7 \mathrm{~mm}$ de largo y $3.5 \mathrm{~mm}$ de ancho $(8.3-9 \times 3.7-4.5 \mathrm{~mm})$; las alas y la quilla 
son también más pequeñas que las descritas por Barneby (1964, p. 167) en aproximadamente la misma proporción. Al momento de colectar las plantas de Durango (Spellenberg and Gonzalez E. 10354) éstas estaban en floración temprana, pero las vainas (NMC) que quedaron de la temporada anterior eran de $7 \mathrm{~mm}$ de diámetro $(5-6 \mathrm{~mm})$; la dehiscencia comienza en la base y continúa hacia el ápice en la mitad proximal de la sutura adaxial y el septo (dehiscencia desconocida); a este respecto la dehiscencia de la vaina se parece mucho a la de su congénere $A$. strigulosus Kunth ex H.B.K. Los ovulos varlan en número de 10 a $18(20-24)$.

Las dos colectas de Durango de $A$. zacatecanus, y muchas otras de $A$. strigulosus que se han hecho desde 1964 en otros lugares en México, indican que A. zacatecanus podría ser incluida como parte de la variación de $A$. strigulosus, esta última considerada como una especie más amplia. Tal opinión ha sido expresada por el Sr. Barneby después de haber revisado un duplicado de la colecta de Chávez, en una carta dirigida a Spellenberg (25 enero 1992, depositada con la muestra Chávez 53 en NMC). Aparentemente el grupo necesita una revisión moderna.

Astragalus goldmanii, A. hartwegii, y A. vaccarum son miembros de Micranthi una pequeña sección con cinco especies, según Barneby. Previamente, sólo se conocía el número cromosómico de un miembro de este grupo ( $A$. clevelandii) Green, $n=13$, Spellenberg, 1976). Los números adicionales presentados aqui apoyan la hipótesis de Barneby, quien considera que $A$. clevelandii está relacionada con las especies mexicanas, muchas de las cuales, incluyendo las de la sección Micranthi, tienen números cromosómicos de $n=14$ y 15 (Spellenberg, 1976). Los conteos de $n=11$ para especies de secciones cuya distribución se ubica principalmente en el oeste de Norteamérica está en concordancia con los patrones citologicos previamente conocidos (Spellenberg, 1976).

\section{AGRADECIMIENTOS}

Agradecemos a Rupert Barneby por su ayuda en la revisión del tipo de $\boldsymbol{A}$. zacatecanus y en la confirmación de la identificación de la especie de la nueva colecta. Extendemos un especial agradecimiento a la Dra. Socorro González Elizondo, quien aportó el transporte y experiencia botánica durante una excursión de varios días a la Reserva de la Biosfera "La Michilla" en Durango. Bradd Shulke, estudiante de New Mexico State University, realizo los conteos para las dos diferentes formas florales de A. longissimus.

\section{LITERATURA CITADA}

Barneby, R.C. 1964. Atlas of North American Astragalus. Mem. New York Bot. Gard. 13 (2 parts): 1-1188. Spellenberg, R. 1976. Chromosome numbers and their cytotaxonomic significance for North American Astragalus (Fabaceae). Taxon 25: 463-476. 